\title{
OPTIMAL DESIGN OF LINEAR ARRAYS IN A TDMA CELLULAR SYSTEM WITH GAUSSIAN INTERFERENCE
}

\author{
S. Savazzi, O. Simeone and U. Spagnolini \\ Dipartimento di Elettronica e Informazione, Politecnico di Milano \\ P.za L. da Vinci, 32, I-20133 Milan, Italy \\ simeone, spagnoli@elet.polimi.it
}

\begin{abstract}
Optimal design of a linear antenna array at the base station is investigated for the uplink of a TDMA cellular system. The problem is formulated as the maximization of the (ergodic or outage) capacity and entails the trade-off between diversity gain (large antenna spacing) and interference-rejection capability of the array (small antenna spacing). In many applications the positions of user and interferers are not known a priori at the time of the antenna deployment or the terminals are mobile. Therefore, the optimization is carried out not only for a fixed positions of users and interferers, but also by averaging the capacity over the positions of users and interferers within their cells.

The approach to the solution of this problem is twofold. At first, an approximate analytical closed form expression for the capacity as a function of the antenna spacing is derived. Then, the results so obtained are corroborated by optimizing the exact capacity though an extensive search over the optimization domain.
\end{abstract}

\section{INTRODUCTION}

Antenna arrays have emerged in the last decade as a powerful technology in order to increase the link or system capacity in wireless systems. Basically, the deployment of multiple antennas at either the transmitter or the receiver side of a wireless link allows the exploitation of diversity and beamforming gains. Diversity relies on fading uncorrelation and provides a powerful means to combat the impairments caused by channel fluctuations [1]. On the other hand, when fading is highly correlated, beamforming can be employed to mitigate the spatially correlated noise (e.g., interference) [2].

Given a propagation environment, the degree of spatial correlation can be controlled by selecting the inter-element spacings of the array. The optimal selection is a trade-off among several conflicting requirements and the analysis in a realistic setting is far from being trivial. In this paper, the analysis is referred (but not necessarily restricted) to the uplink (i.e., mobile-to-base station) of a cellular system, where
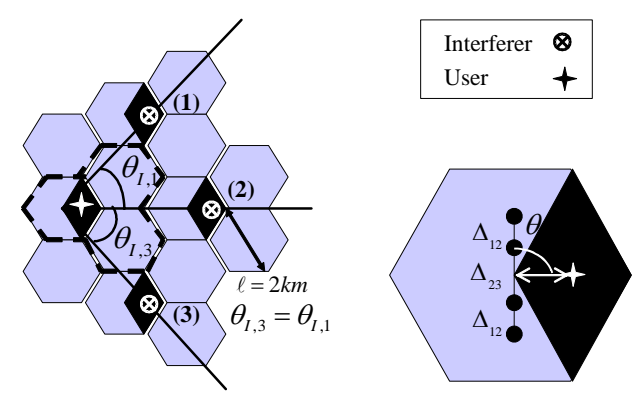

Fig. 1. Interference layout for a cellular system with hexagonal cells and trisectorial antennas at the base stations (reuse factor $F=3$, antenna array with $N=4$ antennas).

the base station is equipped with a linear array and noise arises from inter-cell interference here assumed as Gaussian.

The setting is exemplified in fig. 1 . Therein, we have a cellular system with hexagonal cells and a frequency reuse factor $F=3$ (clusters are denoted by dashed lines). The base station is equipped with an antenna array with $N=4$ antennas for the coverage sector of $60 \mathrm{deg}$. Each terminal is provided with one omindirectional antenna. The assumed propagation environment ranges from line of sight condition to rich scattering according to the ring spatial correlation model [3]. The user of interest is located in the sector shown in the figure and the corresponding first ring of interference is denoted by the shaded cells. The array is symmetric with respect to its middle point in order to reflect the symmetry of the layout at hand. The problem we tackle is the following: find the optimal antenna spacings, in the example the vector $\boldsymbol{\Delta}=\left[\begin{array}{ll}\Delta_{12} & \Delta_{23}\end{array}\right]^{T}$, so that the (either ergodic or outage) capacity of the system is maximized.

Since in many applications the position of users and interferers is not known a priori at the time of the antenna deployment or the in-cell/out-cell terminals are mobile, it is of interest to evaluate the optimal spacings not only for 
a fixed position of users and interferers but also by averaging the capacity over the positions of user and interferers within their cells. Here, the approach to the solution of this problem is twofold. At first, we find an approximate analytical closed form expression for the capacity with respect to the antenna spacing and then optimize this function numerically. Then, the results so obtained are corroborated by optimizing the exact capacity through an extensive search over the optimization domain.

\section{PROBLEM FORMULATION}

The signal received by the base station within the cell of the user of interest can be written as

$$
\mathbf{y}=\mathbf{h} x+\sum_{i=1}^{M} \mathbf{h}_{I, i} x_{I, i}+\mathbf{n},
$$

where $\mathbf{h}$ is the $N \times 1$ vector describing the channel gains between the in-cell user and the $N$ antennas at the base station, $x$ is the signal transmitted by the user, $\mathbf{h}_{I, i}$ and $x_{I, i}$ are the corresponding quantities referred to the $i$ th interferer ( $i=1, \ldots, M)$ and $\mathbf{n}$ is the additive white Gaussian noise with $E\left[\mathbf{n n}^{H}\right]=\sigma^{2} \mathbf{I}$. The channel vectors $\mathbf{h}$ and $\left\{\mathbf{h}_{I, i}\right\}_{i=1}^{M}$ are assumed to be zero-mean complex Gaussian (Rayleigh fading) with spatial correlation $\mathbf{R}=E\left[\mathbf{h h}^{H}\right]$ and $\left\{\mathbf{R}_{I, i}=E\left[\mathbf{h}_{I, i} \mathbf{h}_{I, i}^{H}\right]\right\}_{i=1}^{M}$ respectively, obtained according to the statistical model in [3] and briefly reviewed in Sec. 3.

The instantaneous capacity is

$$
C=\log _{2}\left(1+\mathbf{h}^{H} \mathbf{R}_{n}^{-1} \mathbf{h}\right)[b i t / s / H z]
$$

where $\mathbf{R}_{n}=\mathbf{R}_{I}+\sigma^{2} \mathbf{I}_{N}$ accounts for the spatial covariance matrix of the interferers $\mathbf{R}_{I}=\sum_{i=1}^{M} \mathbf{R}_{I, i}$ and thermal noise. In the following, we will consider an interferencelimited scenario so that $\mathbf{R}_{n} \simeq \mathbf{R}_{I}$. For a fading channel, it is relevant to consider either the ergodic capacity (i.e., the ensemble average $E[C]$ ) or the outage capacity for a given outage probability $p$ (i.e., the value $C_{p}$ such that $P(C \leq$ $\left.C_{p}\right)=p$ ). The ergodic capacity is a performance criterion suited for fast-varying channels or delay-insensitive applications whereas the outage probability is appropriate for channels varying slowly as compared to the length of the coded packet [4]. In the following the performance criterion, either the ergodic or the outage capacity, will be denoted by $\mathcal{L}(\boldsymbol{\Delta}, \boldsymbol{\eta})$. Notice that the notation stresses the dependence of the performance measure on the set of antenna spacing $\boldsymbol{\Delta}$ and the position of the users $\boldsymbol{\eta}=\left[d, d_{I, 1}, \ldots, d_{I, M}\right]^{T}$. More specifically, let the antenna array at the base station be equipped with $N$ antennas belonging to a linear array, to simplify $N$ is an even number of antennas. Denoting by $\Delta_{i j}$ the distance between the $i$ th and the $j$ th element and recalling that we are assuming a symmetric array (with respect to

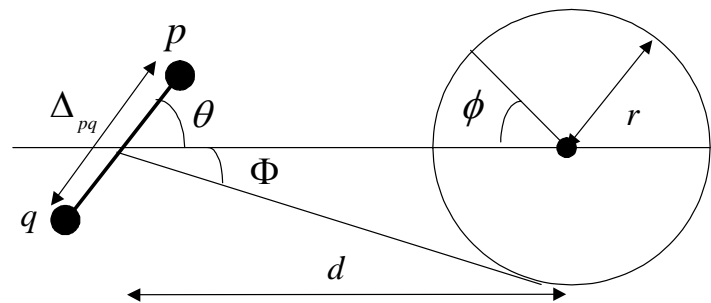

Fig. 2. Ring model for scatterers employed for the computation of the spatial correlation

its middle point), the set of $N / 2 \times 1$ unknowns spacings to be determined is $\boldsymbol{\Delta}=\left[\Delta_{12} \Delta_{23} \cdots \Delta_{N / 2, N / 2+1}\right]^{T}$.

The first problem we tackle is that of finding the set of optimal spacings $\hat{\Delta}$ that maximizes the selected performance criterion $\mathcal{L}(\boldsymbol{\Delta}, \boldsymbol{\eta})$ (either ergodic or outage capacity):

$$
\text { (Problem 1): } \hat{\Delta}=\arg \max _{\boldsymbol{\Delta}} \mathcal{L}(\boldsymbol{\Delta}, \boldsymbol{\eta}) .
$$

Since $\mathcal{L}(\boldsymbol{\Delta}, \boldsymbol{\eta})$ depends on the positions of users and interferers, problem 1 is a suited criterion for fixed system with a known layout at the time of antenna deployment.

In mobile systems or in case the position of users and interferers is not known a priori at the time of the antenna deployment (or cells are asynchronous), it is more relevant to maximize the performance criterion $\mathcal{L}(\boldsymbol{\Delta}, \boldsymbol{\eta})$ averaged over the distribution of the locations of users and interferers, that is assumed to be uniform within the sector. We denote this averaging operation by $\langle\mathcal{L}(\boldsymbol{\Delta}, \boldsymbol{\eta})>$. Therefore, the second problem can be stated as

$$
\text { (Problem 2): } \hat{\Delta}=\arg \max _{\boldsymbol{\Delta}}<\mathcal{L}(\boldsymbol{\Delta}, \boldsymbol{\eta})>
$$

\section{SPATIAL CORRELATION MODEL}

The spatial model considered in this work is illustrated in fig. 2. The single antenna user is surrounded by a ring of scatterers of radius $r$. The antennas at the base station receive the signal scattered by the ring through a narrow angular spread $\Phi \simeq r / d$, where $d$ is the distance between the base station and the user. Different propagation environments are modelled by either varying the distribution of scatterers along the ring or the angular spread at the base station $\Phi$. In particular, a small $\Phi$ implies a line of sight channel whereas increasing $\Phi$ models a rich scattering condition.

The normalized spatial correlation $\left[\mathbf{R}^{\prime}\right]_{p, q}$ between the channel gains measured at the $p$ th and the $q$ th antenna of the base station can be evaluated according to the geometric 
model [3] for a uniform distribution of the scatterers along the ring as:

$$
\left[\mathbf{R}^{\prime}\right]_{p, q}=\exp \left[j \frac{2 \pi}{\lambda} \Delta_{p q} \cos (\theta)\right] J_{0}\left(\frac{2 \pi}{\lambda} \Delta_{p q} \Phi \sin (\theta)\right),
$$

$\lambda$ is the wavelength. For a path loss exponent $\alpha$, the spatial correlation matrix $\mathbf{R}$ of the user at distance $d$ is $\mathbf{R}=$ $\left(d_{r e f} / d\right)^{\alpha} \cdot \mathbf{R}^{\prime}$, where $d_{r e f}$ is a reference distance.

The ring model is assumed to hold for the interferers as well, possibly with different geometric characteristics. The subscript $I$ denotes the quantities defined above for the interferers. Therefore, the channel of the $i$ th interferer is characterized by a distance $d_{I, i}$, an angular spread $\Phi_{I, i}$ and an angle $\theta_{I, i}$. The corresponding normalized correlation matrix for the $i$ th user reads $\mathbf{R}_{I, i}^{\prime}$ and is obtained according to (4). The interference correlation matrix is finally: $\mathbf{R}_{I}=\sum_{k=1}^{M}\left(\frac{d_{r e f}}{d_{I, i}}\right)^{\alpha} \mathbf{R}_{I, i}^{\prime}$.

\section{ANALYTICAL EXPRESSION FOR THE PERFORMANCE CRITERION}

In this section we propose an approximation for the spatial correlation matrix $\mathbf{R}$ to simplify the performance criterion $\mathcal{L}(\boldsymbol{\Delta}, \boldsymbol{\eta})$ for analytical derivations. In Sec. 4.1 we consider the case where the angular spread for users and interferers is small so that a rank- 1 approximation of the spatial correlation matrices can be used. Generalization is given in Sec. 4.2.

\subsection{Rank-1 approximation}

If the angular spread is small (i.e., $\Phi \ll 1$ ) the spatial covariance matrices of user and interferers, $\mathbf{R}$ and $\left\{\mathbf{R}_{I, i}\right\}_{i=1}^{M}$, can be approximated by rank-1 matrices. Let us consider for instance the correlation matrix $\mathbf{R}$ for the user. For $\Phi \ll 1$ the term $J_{0}\left(\frac{2 \pi}{\lambda} \Delta_{p q} \Phi \sin (\theta)\right) \simeq 1$ can be simplified in (4) so that the following decomposition holds

$$
\mathbf{R} \simeq \rho \cdot \mathbf{q q}^{H}
$$

where $\rho=\left(d_{\text {ref }} / d\right)^{\alpha}$ and

$\left.\mathbf{q}=\left[\begin{array}{llll}1 & \exp \left[-j \omega(\theta) \Delta_{12}\right. & \cdots & \exp \left[-j \omega(\theta) \Delta_{1 N}\right.\end{array}\right]\right]^{T}$

for $\omega(\theta)=2 \pi / \lambda \sin (\theta)$. The same holds for the correlation matrices of interferers: $\mathbf{R}_{I, i} \simeq \rho_{I, i} \mathbf{q}_{I, i} \mathbf{q}_{I, i}^{H}$, where $\rho_{I, i}$ and the vector $\mathbf{q}_{I, i}$ are selected as explained above.

From (5), the channel vector can be written as $\mathbf{h}=$ $\gamma \sqrt{\rho} \mathbf{q}$ where $\gamma \sim \mathcal{C N}(0,1)$. Therefore, within the rank1 approximation, the instantaneous capacity (1) reads

$$
C=\log _{2}\left(1+|\gamma|^{2} \rho \mathbf{q}^{H}\left(\sum_{i=1}^{M} \rho_{I, i} \mathbf{q}_{I, i} \mathbf{q}_{I, i}^{H}\right)^{-1} \mathbf{q}\right) .
$$

Since the logarithm is a monotone increasing function and the parameters $\gamma$ and $\rho$ do not depend on the antenna spacing $\boldsymbol{\Delta}$, the instantaneous capacity is maximized (as a function of $\boldsymbol{\Delta})$ if the argument $\mathbf{q}^{H}\left(\sum_{i=1}^{M} \rho_{I, i} \mathbf{q}_{I, i} \mathbf{q}_{I, i}^{H}\right)^{-1} \mathbf{q}$ is maximized. Notice that optimizing the instantaneous capacity entails the maximization of both the ergodic and outage probability so that the performance criterion $\mathcal{L}(\boldsymbol{\Delta}, \boldsymbol{\eta})$ in this Section denotes indifferently either quantity. Now, the optimization in problem 1 (2) can be stated as

$$
\hat{\boldsymbol{\Delta}}=\arg \min _{\boldsymbol{\Delta}} \mathcal{L}^{\prime}(\boldsymbol{\Delta}, \boldsymbol{\eta}),
$$

where $\mathcal{L}^{\prime}(\boldsymbol{\Delta}, \boldsymbol{\eta})=\mathbf{q}^{H}\left(\sum_{i=1}^{M} \rho_{I, i} \mathbf{q}_{I, i} \mathbf{q}_{I, i}^{H}\right) \mathbf{q}$. After tedious but straightforward algebraic computations the performance criterion can be reduced to

$$
\begin{aligned}
\mathcal{L}^{\prime}(\boldsymbol{\Delta}, \boldsymbol{\eta})= & N M+\sum_{i=1}^{M}\left(\frac{d_{r e f}}{d_{i}}\right)^{\alpha}\left[\sum_{j=1}^{L} 4 S\left(l_{j}, \theta_{1}, \theta_{I, i}\right)\right. \\
& \left.+\sum_{k=1}^{C} 2 S\left(c_{k}, \theta, \theta_{I, i}\right)\right]
\end{aligned}
$$

where $S\left(x, \theta_{1}, \theta_{2}\right)=\cos \left[2 \pi x\left(\sin \left(\theta_{1}\right)-\sin \left(\theta_{2}\right)\right)\right], L=$ $\left(\left(\begin{array}{c}N \\ 2\end{array}\right)-\frac{N}{2}\right) / 2$ is the number of "lateral spacings" $l_{i}=\Delta_{i, j}$ for $i=1, \ldots, \frac{N}{2}-1, j=i+1, \ldots, N-i, C=N / 2$ is the number of "central spacings" $c_{i}=\Delta_{i, N-i}$ for $i=$ $1, \ldots, N / 2$.

The minimization of $\mathcal{L}^{\prime}(\boldsymbol{\Delta}, \boldsymbol{\eta})$ (9) can be obtained by standard techniques. However, it is interesting to consider from (8) that if we can find a set of antenna spacing $\tilde{\Delta}$ such that the user vector $\mathbf{q}$ is orthogonal to all the $M$ interference vectors $\mathbf{q}_{I, i}$ then $\mathcal{L}^{\prime}(\tilde{\Delta}, \boldsymbol{\eta})=0$, which implies that $\tilde{\Delta}$ is a solution of the problem at hand (2), i.e., $\hat{\boldsymbol{\Delta}}=\tilde{\boldsymbol{\Delta}}$.

The solution of Problem-2 (3) by means of the analysis presented in this Section is not directly feasible. However, using the Jensen's inequality, it is straightforward to show that the maximization of an upper bound on $\mathcal{L}(\boldsymbol{\Delta}, \boldsymbol{\eta})$ can be obtained by solving $\hat{\boldsymbol{\Delta}}=\arg \min _{\boldsymbol{\Delta}}\left\langle\mathcal{L}^{\prime}(\boldsymbol{\Delta}, \boldsymbol{\eta})\right\rangle$, where the average $\left\langle\mathcal{L}^{\prime}(\boldsymbol{\Delta}, \boldsymbol{\eta})>\right.$ has to be evaluated numerically.

\subsection{Rank $>1$ approximation}

When the angular spread $\Phi$ becomes larger, the rank-1 approximation of the correlation matrix (5) may not be acceptable. In this case we can resort a rank- $a$ approximation with $a>1$ :

$$
\mathbf{R} \simeq \sum_{k=1}^{a} \rho^{(k)} \cdot \mathbf{q}^{(k)} \mathbf{q}^{(k) H},
$$

the set of vectors $\left\{\mathbf{q}^{(k)}\right\}_{k=1}^{a}$ are required to be linearly independent (the reasoning is similar for $\mathbf{R}_{I, i}$ ). In this paper we limit the analysis to the case $a=2$. The expression of vector $\mathbf{q}^{(k)}$ from (4) with respect to the antenna spacings is 
not trivial as in the rank-1. However, in analogy with (6), we could set

$$
\left.\mathbf{q}^{(k)}=\left[\begin{array}{llll}
1 & \exp \left[-j \omega^{(k)} \Delta_{12}\right.
\end{array}\right] \quad \cdots \quad \exp \left[-j \omega^{(k)} \Delta_{1 N}\right]\right]^{T},
$$

where the wavenumbers $\left(\omega^{(1)}, \omega^{(2)}\right)$ have to be determined according to some criterion. In order to be consistent with the rank- 1 case considered in the previous Section, we try to minimize the Frobenius norm of approximation error relative to (10). It can be proved that this results in

$$
\omega^{(1)}=\omega(\theta)+\varphi, \omega^{(2)}=\omega(\theta)-\varphi
$$

with $\varphi=2 \pi / \lambda \cdot \Phi \cos (\theta)$ and $\rho^{(1)}=\rho^{(2)}=\left(d_{\text {ref }} / d\right)^{\alpha} / 2$. For each interferer, the discussion above applies by appropriately modifying equations (10)-(11) and (12).

Differently from the rank-1 case where the problem (2) was solved for both ergodic and outage capacity, here we focus on the ergodic capacity $E[C]$ and optimize an upper bound on $E[C]$ directly derived from the Jensen's inequality:

$$
\begin{aligned}
E[C] & =E\left[\log _{2}\left(1+\mathbf{h}^{H} \mathbf{R}_{I}^{-1} \mathbf{h}\right)\right] \\
& \leq \log _{2}\left(1+E\left[\mathbf{h}^{H} \mathbf{R}_{I}^{-1} \mathbf{h}\right]\right)
\end{aligned}
$$

The limits of this approach will be assessed by numerical simulation in Sec. 5. The problem can then be stated as

$$
\hat{\boldsymbol{\Delta}}=\arg \max _{\boldsymbol{\Delta}} E\left[\mathbf{h}^{H} \mathbf{R}_{I}^{-1} \mathbf{h}\right]=\arg \min _{\boldsymbol{\Delta}} E\left[\mathbf{h}^{H} \mathbf{R}_{I} \mathbf{h}\right] .
$$

By defining $\mathcal{L}^{\prime \prime}(\boldsymbol{\Delta}, \boldsymbol{\eta})=E\left[\mathbf{h}^{H} \mathbf{R}_{I} \mathbf{h}\right]$ and after some algebraic manipulations it simplifies as

$$
\begin{gathered}
\mathcal{L}^{\prime \prime}(\boldsymbol{\Delta}, \boldsymbol{\eta})=N M+\sum_{i=1}^{M}\left(\frac{d_{r e f}}{d^{(i)}}\right)^{\alpha}\left[\sum_{j=1}^{L} 4 S\left(l_{j}, \theta, \theta_{I, i}\right)(15)\right. \\
\left.\cdot T_{i}\left(l_{j}\right)+\sum_{k=1}^{C} 2 S\left(c_{k}, \theta, \theta_{I, i}\right) T_{i}\left(c_{k}\right)\right],
\end{gathered}
$$

where $T_{i}(x)=\cos (\varphi x) \cos \left(\varphi_{I, i} x\right)$.

Similarly to the previous Section, an approximate solution to problem (3), that has to be validated by numerical simulations (see Sec. 5), is that of solving

$$
\hat{\Delta}=\arg \min _{\boldsymbol{\Delta}}<\mathcal{L}^{\prime \prime}(\boldsymbol{\Delta}, \boldsymbol{\eta})>\text {. }
$$

\section{NUMERICAL RESULTS}

In this Section we compare the solution of problems (2)-(3) obtained by optimizing the analytical expression of the capacity derived in the previous Section with the optimization the exact capacity carried out through an extensive search over the optimization domain. Here we consider the layout in fig. $1(N=4, M=3, F=3$ ) with cell diameter

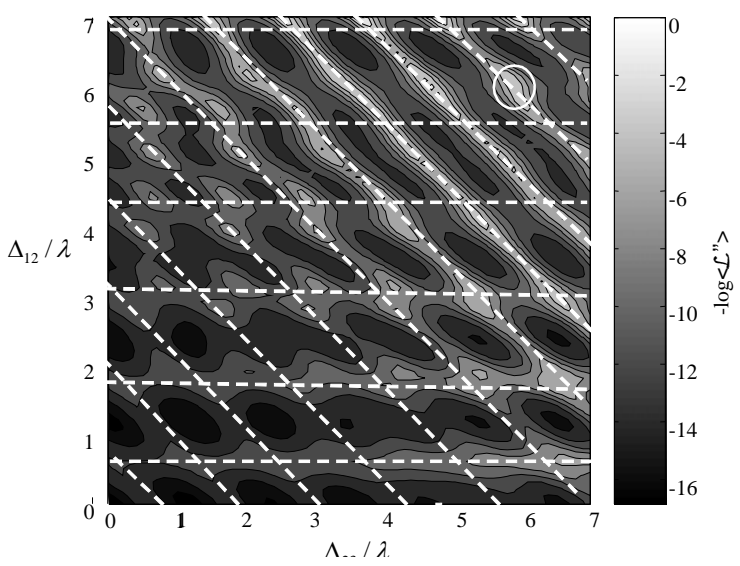

Fig. 3. Rank-2 approximation $\mathcal{L}^{\prime \prime}(\boldsymbol{\Delta}, \boldsymbol{\eta})(15)$ versus $\Delta_{12} / \lambda$ and $\Delta_{23} / \lambda$.Dashed lines represent the optimality conditions (17a)-(17b) obtained by the rank-1 approximation.

$\ell=2 \mathrm{~km}$. The channels of user and interferers are assumed to be characterized by the same scatterer radius $r=r_{I, i}$, $i=1, \ldots, M$. At first, we consider the problem (2), where user and interferers are located at the center of their respective sectors (as in fig. 1). Notice that in this case the user is always aligned with the second interferer so that the array has no interference-rejection capability with respect to it.

By minimizing the rank-1 analytical approximation $\mathcal{L}^{\prime}(\boldsymbol{\Delta}, \boldsymbol{\eta})$ (9) that holds for $\Phi \ll 1$, it is found that the optimal spacings guarantee that the channel vector of the user is orthogonal to the channel vectors of the first and third interferer (the second is aligned). To be specific, we get the following conditions $(k=0,1, \ldots)$ :

$$
\begin{aligned}
\hat{\Delta}_{12} & =(2 k+1) \Psi \text { with any } \hat{\Delta}_{23} \geq 0 \text { or } \\
\hat{\Delta}_{12}+\hat{\Delta}_{23} & =(2 k+1) \Psi
\end{aligned}
$$

where $\Psi=\lambda /\left(2 \sin \left(\theta_{I, 1}\right)\right) \simeq 0.61 \lambda$. The set of optimal spacings satisfying (17a)-(17b) is shown in fig. 3 as dashed lines. Notice that according to this solution, that assumes a line of sight channel ( $\Phi \ll 1$ ), there is no advantage of deploying more than two antennas. In fact, the optimality conditions (17a)-(17b) are met by setting either $\hat{\Delta}_{23}=0$, which corresponds to have three antennas, or $\hat{\Delta}_{12}=0$, which entails the use of two antennas. It can be shown that this result is confirmed (not shown here) by performing an exact optimization of both the ergodic and outage capacity with $r<1 m$ (i.e., $\Phi<0.1 \mathrm{deg}$, not shown).

Let us now consider the rank $a=2$ approximation. Fig. 3 shows $-\log \mathcal{L}^{\prime \prime}(\boldsymbol{\Delta}, \boldsymbol{\eta})$ (15) versus $\Delta_{12}$ and $\Delta_{23}$. As it is clear, in case the channel is not purely line of sight, the antennas should be deployed in such a way to guarantee a satisfactory degree of diversity. Therefore, the optimal spacings still satisfy conditions (17a)-(17b), but among these so- 


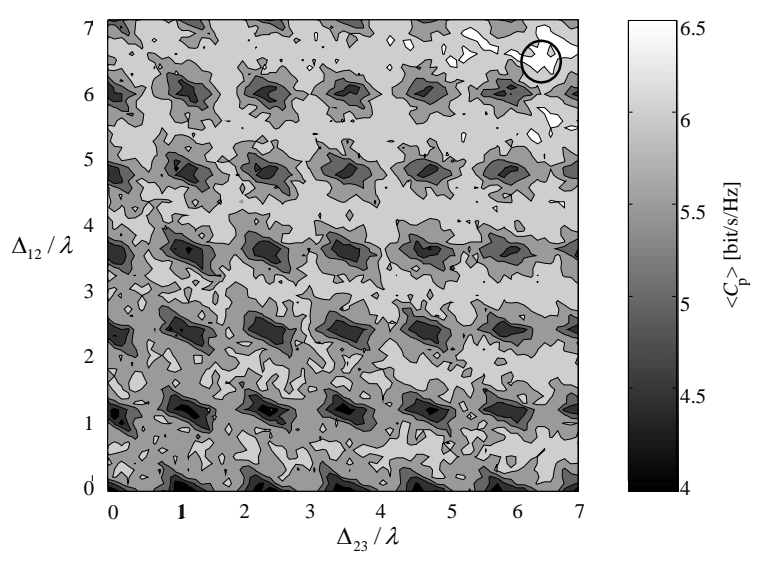

Fig. 4. Outage capacity $C_{0.1}[\mathrm{bit} / \mathrm{s} / \mathrm{Hz}]$ versus $\Delta_{12} / \lambda$ and $\Delta_{23} / \lambda(r=50 m)$.

lutions, one has to prefer spacings large enough to enforce the property discussed above. For our example, it turns out that this further condition can be stated (approximately) as $\left(\hat{\Delta}_{12}, \hat{\Delta}_{23}\right) \geq 10 \Psi$. Therefore, in order to keep the area occupied by the array as small as possible without sacrificing the performance of the system, it would be more appropriate to select $\hat{\Delta}_{12}=\hat{\Delta}_{23}=10 \Psi \simeq 6.1 \lambda$ (marked by a circle in fig. 3). This conclusion is confirmed by the exact outage capacity with $p=0.1$ in fig. 4 for $r=50 \mathrm{~m}$ ( $\Phi=5.7 \mathrm{deg}$ ). In particular, optimizing the antenna spacings yields a performance benefits that amounts to approximately $4 \mathrm{bit} / \mathrm{s} / \mathrm{Hz}$ as compared to the conventional adaptive arrays with $\hat{\Delta}_{12}=\hat{\Delta}_{23}=\lambda / 2$. Notice that even though $\mathcal{L}^{\prime \prime}(\boldsymbol{\Delta}, \boldsymbol{\eta})$ was obtained in Sec. 4.2 as an approximation of the ergodic capacity, the conclusion turned out to be valid even for the maximization of the outage capacity.

Let us now turn to the solution of problem (3). In this case, the optimal set of spacings $\hat{\Delta}$ should guarantee the best performance on average with respect to the positions of user and interferers within the corresponding sectors. Our analysis shows that for the layout at hand, the (average) performance gain with respect to the conventional adaptive arrays with $\hat{\Delta}_{12}=\hat{\Delta}_{23}=\lambda / 2$ has decreased as compared to the fixed case studied above. This result is confirmed by computing the exact outage capacity $\left\langle C_{p}\right\rangle$ with $p=0.1$ for $r=50 \mathrm{~m}$. In fig. 5, the approximation $-\log <\mathcal{L}^{\prime \prime}(\boldsymbol{\Delta}, \boldsymbol{\eta})>$ (lower) and the capacity $<C_{0.1}>$ are plotted versus $\Delta_{12} / \lambda=\Delta_{23} / \lambda$. Selecting the optimal value now yields a performance benefit that is around $2 b i t / s / H z$ in capacity.
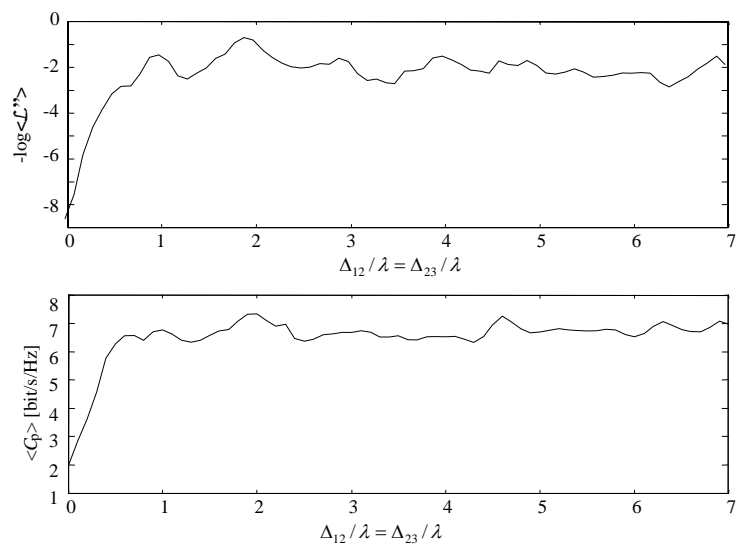

Fig. 5. Rank-2 approximation $\mathcal{L}^{\prime \prime}(\boldsymbol{\Delta}, \boldsymbol{\eta})$ (upper figure) and outage capacity $\left\langle C_{0.1}\right\rangle$ (lower figure) averaged over the positions of user and interferers versus $\Delta_{12} / \lambda=\Delta_{23} / \lambda$.

\section{CONCLUSION}

The optimal antenna spacings for a linear array at the base station of a cellular TDMA system have been investigated by obtaining a closed form expression for a performance criterion related to the maximization of the (ergodic or outage) capacity. The results obtained through the analysis have been validated through comparison by an exhaustive search over the optimization domain for the exact capacity, showing the effectiveness of the analytical approach.

\section{REFERENCES}

[1] G. J. Foschini and M. J. Gans, "On limits of wireless communications in a fading environment when using multiple antennas,” Wireless Personal Communications, Vol. 6, No. 3, pp. 311-335, March 1998.

[2] F. Rashid-Farrokhi, K.J.R. Liu and L. Tassiulas, "Transmit beamforming and power control for cellular wireless systems," IEEE Selected Areas in Commun., vol. 16, no. 8, pp. 1437-1450, Oct. 1998.

[3] A. Abdi and M. Kaveh, "A space-time correlation model for multielement antenna systems in mobile fading channels”, IEEE J. Select. Areas Commun., vol. 20, no. 3, pp. 550-560, April 2002.

[4] E. Biglieri, J. Proakis and S. Shamai, "Fading channels: information-theoretic and communications aspects," IEEE Trans. Inform. Theory, vol. 44, no. 6, pp. 2619-2692, Oct. 1998. 